\title{
Radiative-lifetime measurements and calculations of odd-parity highly excited levels in Ba I
}

\author{
Wei Zhang, ${ }^{1,2}$ Patrick Palmeri, ${ }^{3}$ Pascal Quinet, ${ }^{3,4}$ Émile Biémont, ${ }^{3,4, *}$ Shan Du, ${ }^{1,2}$ and Zhenwen Dai ${ }^{1,2,5, \dagger}$ \\ ${ }^{1}$ College of Physics, Jilin University, Changchun 130021, China \\ ${ }^{2}$ Key Lab of Coherent Light, Atomic and Molecular Spectroscopy, Ministry of Education, Changchun 130021, China \\ ${ }^{3}$ Astrophysique et Spectroscopie, Université de Mons, B-7000 Mons, Belgium \\ ${ }^{4}$ IPNAS (Bât. B15), Université de Liège, Sart Tilman, B-4000 Liège, Belgium \\ ${ }^{5}$ Institute of Atomic and Molecular Physics, Jilin University, Changchun 130012, China
}

(Received 11 September 2010; published 14 October 2010)

\begin{abstract}
Natural radiative lifetime measurements have been performed for 70 odd-parity highly excited levels of neutral barium in the energy range from 30815.512 to $41759.93 \mathrm{~cm}^{-1}$ by a time-resolved laser-induced fluorescence technique in a laser-produced plasma. The lifetime values measured in this paper are in the range from 11.3 to $901 \mathrm{~ns}$. They are compared with the published lifetimes of four levels. Two of them are in good agreement, whereas for the other two our measurements are slightly longer than the published data. The reasons for the discrepancies are discussed. Comparisons with theoretical results of the Hartree-Fock method with relativistic corrections illustrate the difficulties associated with the use of Cowan's codes for obtaining accurate branching fractions for transitions depopulating highly excited levels along the Rydberg series of heavy neutral elements. This work will be useful to extend the set of oscillator strengths available in Ba I.
\end{abstract}

DOI: 10.1103/PhysRevA.82.042507

PACS number(s): 32.70.Cs, 42.62.Fi, 31.15.-p

\section{INTRODUCTION}

In traditional electronic engineering, barium was initially used as a getter in electro-vacuum devices, as an important component of high-temperature superconductors [1], and, in recent years, as an active medium in gas lasers [2]. The study of the radiative properties of the barium atom (radiative lifetimes, transition probabilities, branching fractions, etc.) is therefore very important. In addition, it is well known that the combination of accurate radiative lifetimes with precise branching fractions provides the best experimental approach for the determination of absolute transition probabilities, because these quantities are crucial parameters in the determination of solar and stellar abundances [3].

In fact, the study of the energy levels [4-11] and transition probabilities [12] of Ba I has a long history. Experimental measurements of radiative lifetimes for some even-parity levels [13-21] and calculations for the $6 s n f^{3} F_{2}(11 \leqslant n \leqslant 35)$ Rydberg series and some $6 s 6 p, 6 s 7 p, 5 d 5 p$, and $5 d 6 p$ levels $[22,23]$ have been reported. But because it is difficult or impossible to excite most of the high-lying odd-parity levels from the ground level ${ }^{1} S_{0}$ and because the lowest metastable levels $6 s 5 d^{3} D_{J}(J=1$ to 3$)$ located above $9000 \mathrm{~cm}^{-1}$ are difficult to populate in a thermal atomic beam, direct lifetime measurements for such levels are scarce. The previous investigations of radiative lifetimes for odd-parity levels were limited to a few low-lying levels. The lifetime of the $6 s 6 p^{1} P_{1}^{\circ}$ level of Ba I was measured by Hulpke et al. [24], while the lifetime of the same level was obtained by Lurio et al. [25] using the zero-field level-crossing (Hanle) method. Dickie et al. [26-28] investigated the lifetimes of the $6 s 6 p^{1} P_{1}^{\circ}$, $6 s 7 p^{1} P_{1}^{\circ}$, and $5 d 6 p^{1} P_{1}^{\circ}$ levels using the Hanle effect method. By the Hanle effect and optical double resonance, Brecht et al. [29] measured the lifetimes of the $6 s 7 p^{1} P_{1}^{\circ},{ }^{3} P_{1}^{\circ}$, and

\footnotetext{
*e.biemont@ulg.ac.be

${ }^{\dagger}$ dai@jlu.edu.cn
}

$5 d 6 p^{1} P_{1}^{\circ},{ }^{3} P_{1}^{\circ}$, and ${ }^{3} D_{1}^{\circ}$ levels of Ba I. Using the delayedcoincidence technique, the lifetime of the $5 d 6 p^{1} F_{3}^{\circ}$ level has been considered by Jessop et al. [30]. In the investigation of Brink et al. [31], the radiative lifetime of the $5 d 6 p^{3} D_{3}^{\circ}$ level has been measured using the Hanle effect technique. Through consideration of the super-radiance population of the excited levels, the lifetimes of $6 s 7 f^{1} F_{3}^{\circ}$ and $6 s 8 f^{1} F_{3}^{\circ}$ have been obtained by Leonard et al. [18]. The lifetime of $6 s 7 p^{1} P_{1}^{\circ}$ has been investigated by Smedley et al. using the time-resolved laser-induced fluorescence (LIF) technique [19], while, with the same technique, the lifetimes of the $5 d 6 p$ levels of Ba I have been measured by Matsuo et al. [20]. To our best knowledge, no other works on the lifetime data of odd parity levels of $\mathrm{Ba}$ I exist in the literature.

In this paper, by the LIF method, the lifetimes of 70 odd-parity levels, most of which are not accessible by singlephoton excitation from the ground level, are measured through excitation via metastable levels in laser-induced plasma.

\section{EXPERIMENTAL SETUP}

The experimental setup used for lifetime measurements is shown in Fig. 1. In order to produce sufficient atoms in the metastable levels, plasma produced by laser ablation on a $\mathrm{Ba}$ sample has been used as the atomic source. This technique appears to be efficient and reliable for lifetime measurements [32,33]. A 5-mJ 532-nm laser pulse, with about 8 ns duration, from a Q-switched Nd:YAG laser (Continuum Precision II) working at a $10-\mathrm{Hz}$ repetition rate, was focused on the Ba sample by a $30-\mathrm{cm}$-focal-length lens. To ensure that the ablation position is different for the successive pulses, the sample was rotated. A linearly polarized dye laser (Sirah Cobra-Stretch) operating with DCM and Rhodamine B dyes pumped by a Q-switched Nd:YAG 355-nm laser (SpectraPhysics Quanta-Ray), with a $10-\mathrm{Hz}$ repetition rate and about $8 \mathrm{~ns}$ pulse duration, was used for the excitation. The linewidth of the dye laser was about $0.08 \mathrm{~cm}^{-1}$. The second harmonic 


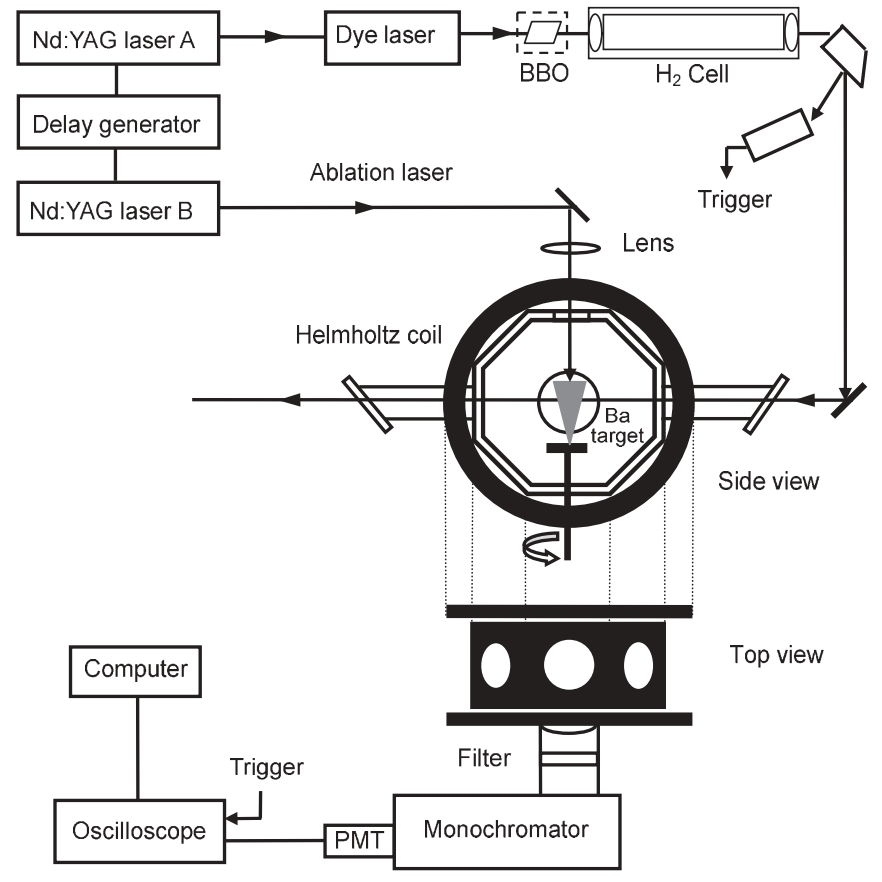

FIG. 1. Experimental setup used for lifetime measurements.

of the dye laser was produced by a $\beta$-barium borate (BBO) type I crystal and then was focused into a $\mathrm{H}_{2}$ gas cell to obtain the different-order Stokes-Raman-shifted components for a tunable uv radiation from 307 to $431 \mathrm{~nm}$. The excitation laser, with pulses of about 5-7 ns duration, was horizontally sent into the vacuum chamber to intersect the vertical atomic beam about $10 \mathrm{~mm}$ above the sample. A digital delay generator (SRS Model 535) was used to adjust the delay between the excitation and ablation pulses. In the direction perpendicular to the laser and the atomic beams, LIF emissions were collected by a fused-silica lens into a grating monochromator for dispersion and then detection by a photomultiplier tube (PMT; Hamamatsu R3896). To enhance the fluorescence collection efficiency, the monochromator was moved by a rotation of $90^{\circ}$ so that its entrance slit was horizontal and parallel to the excitation beam and, in this case, the entrance slit had to be properly widened to avoid the flight-out-of-view effect. A 500-MHz digital oscilloscope (Tektronix TDS 620B), connected to a computer for signal storage and analysis, was used to register the time-resolved photocurrent signal from the detector. In the direction parallel to the horizontal component of the Earth's magnetic field, an appropriate magnetic field of 100 gauss, produced by a pair of Helmholtz coils, was used to wash out the possible decay signal distortion generated by both the recombination background [34] and the quantum beats induced [35] by the Earth's magnetic field.

\section{LIFETIME MEASUREMENTS}

In the present experiment, the interesting highly excited odd-parity levels of $\mathrm{Ba}$ I could be populated from the ground state and from some metastable states through single-photon

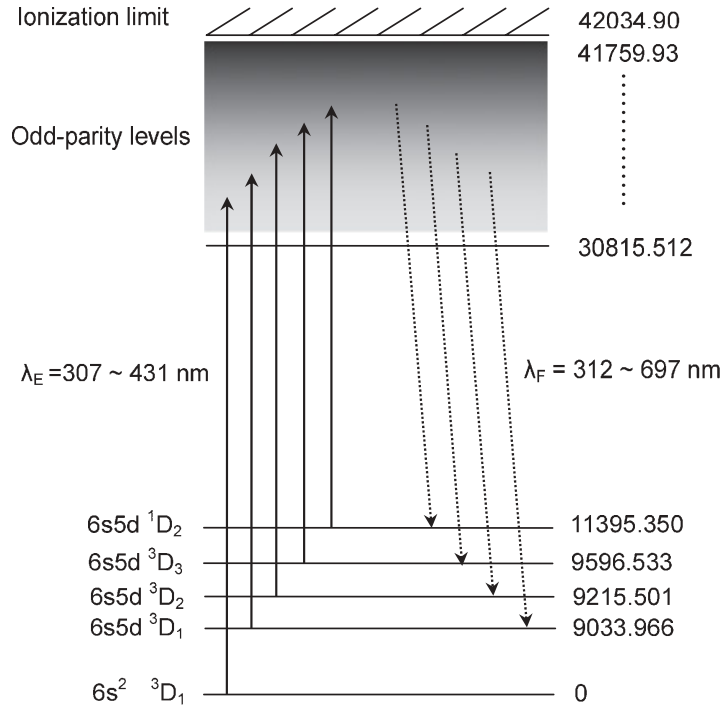

FIG. 2. Partial energy-level diagram of $\mathrm{Ba}$ atom and excitation schemes. The solid lines indicate excitation pathways and the dashed lines indicate fluorescence channels. Energy levels are given in $\mathrm{cm}^{-1}$.

transitions. The diagram of the energy levels and the excitation schemes relevant to the experiment is shown in Fig. 2.

During the measurements, when the delays between the excitation and ablation pulses were varied from 10 to $75 \mu \mathrm{s}$, the detected fluorescence intensities were changed by a factor of 5-10, but the evaluated lifetimes remained constant within the experimental errors. During this process, the speed and density of the atoms in the excitation region gradually decreased and thus the possible perturbing effects, such as radiation trapping, super-radiance, PMT nonlinear response, collision-induced quenching, as well as the flight-out-of-view effect can be effectively checked and eventually eliminated. For the levels with lifetimes longer than $200 \mathrm{~ns}$, more caution should be exercised for avoiding the flight-out-of-view effect. Therefore, for such levels, the delay time was set longer than $25 \mu$ s for obtaining lower-speed atoms. Based on the fact that the atoms in the excitation region traveled $10 \mathrm{~mm}$ in $25 \mu \mathrm{s}$, we deduced that the atomic speed was about $400 \mathrm{~m} / \mathrm{s}$. In order to observe the entire decay process of a level, an observation period of five times the radiative lifetime is needed. For the longest lifetime, close to $1 \mu$ s measured in this paper, during the observation time, Ba atoms flew $2 \mathrm{~mm}$ in the vertical direction. Considering the diameter of about $1 \mathrm{~mm}$ of the exciting laser beam and the imaging character of the fluorescence collective system, a slit width of about $4 \mathrm{~mm}$ has been used in experiments. With this slit width and with a properly increasing delay time, the fight-out-of-view effects can be effectively eliminated. The possible collision with remnant gas was checked by changing the air pressure in the chamber from $2 \times 10^{-4}$ to $3 \times 10^{-3} \mathrm{~Pa}$, while the flight-outof-view effect was checked again by changing the slit width and the position of the monochromator along the propagating direction of the atomic beam. In addition, the effects of radiation trapping, nonlinear response of the PMT detector, and super-radiation have been analyzed and eliminated by varying the pulse energies of the ablation and excitation lasers; the 


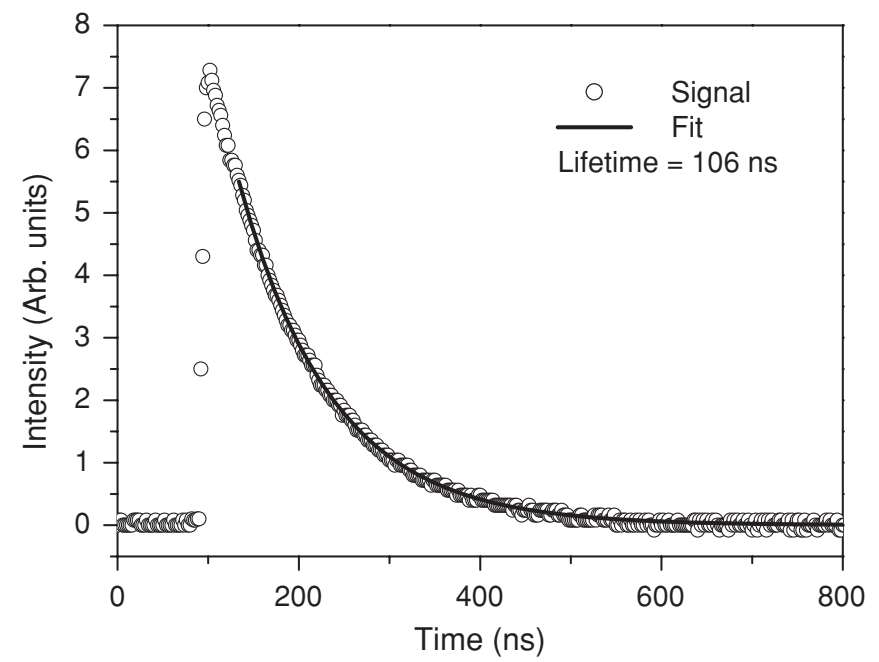

FIG. 3. Typical fluorescence decay curve of the $5 d 7 p^{3} D_{1}^{\circ}$ level with an exponential fitting for lifetime evaluation.

delay time between them was also chosen at an appropriate value.

For each fluorescence decay curve, more than 1000 shots were averaged to acquire a good signal. For a level with a lifetime longer than $70 \mathrm{~ns}$, which is about 10 times the excitation pulse duration, the lifetime value was evaluated by a least-squares exponential fitting to the recorded decay curves. However, we had to be cautious when choosing the starting point of the fitting procedure in order to avoid the stray light of the excitation laser. A typical fluorescence decay curve of the $5 d 7 p^{3} D_{1}^{\circ}$ level with an exponential fitting is shown in Fig. 3. For the levels with shorter lifetimes, the lifetimes were evaluated by a convolution fit of a pure exponential and a laser pulse recorded by the same detection system [36]. A typical fluorescence decay curve for the $5 d 8 p^{3} P_{2}^{\circ}$ level together with a fitted convolution curve is shown in Fig. 4.

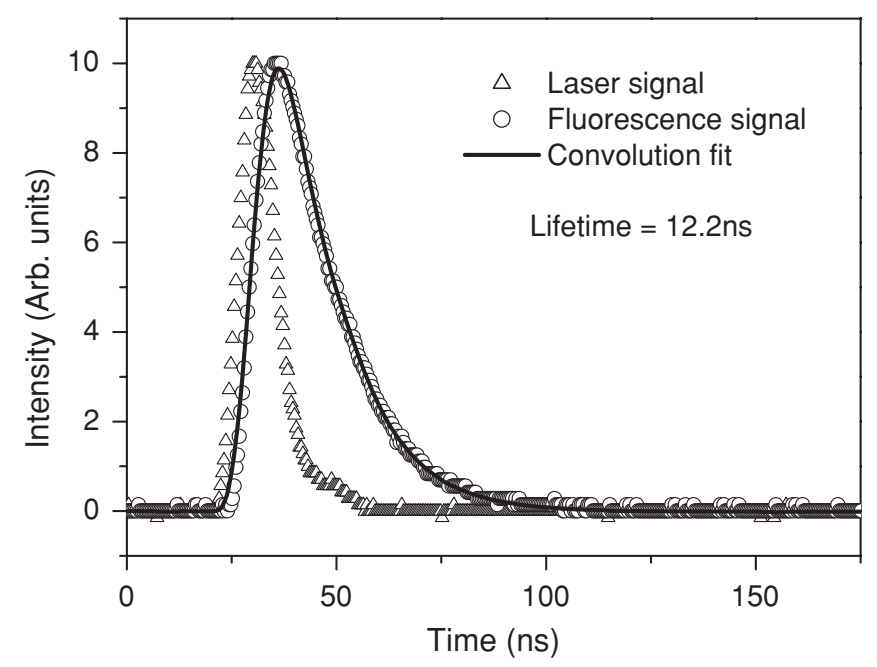

FIG. 4. Fluorescence decay curve of the $5 d 8 p^{3} P_{2}^{\circ}$ level with the fitted convolution curve between the laser pulse and an exponential with a decay constant of $12.2 \mathrm{~ns}$.
In the least-squares exponential fittings, for each fluorescence curve, two lifetime values were determined with two starting points, respectively. One, with the strongest intensity, was chosen at a position outside the region affected by the stray light of the excitation laser, and the other was at a position with half the intensity of the former point. In the convolution procedure, two lifetime values were also evaluated using two excitation pulses recorded before and after the registration of the corresponding fluorescence signal, respectively. For a given curve, the two lifetimes are in close agreement, the remaining differences being mainly due to some systematic effects on both fluorescence curves and exciting pulse shapes including the transit time jitter of the PMT $(1.2 \mathrm{~ns})$, the intensity nonlinear response of the whole detection system, and the randomicity of the stray light of the excitation laser when registering the excitation pulses. The mean value of the two lifetimes and its standard deviation were taken to be the radiative lifetime revealed by the curve and the corresponding systematic error, respectively. For each level, at least 20 curves were recorded under different experimental conditions. The average values of the lifetimes and the systematic errors evaluated from the curves recorded after a sufficiently long delay from the ablation pulse were adopted as the final lifetimes and their systematic errors, respectively.

\section{EXPERIMENTAL RESULTS AND DISCUSSION}

The measured lifetimes of Ba I for the odd-parity levels $6 s n p{ }^{3} P_{1}^{\circ}(n=7-12),{ }^{1} P_{1}^{\circ}(n=7-9,11-14),{ }^{3} P_{0}^{\circ}(n=8,11)$, ${ }^{3} P_{2}^{\circ}(n=8,11-12,14), 6 s n f^{3} F_{2}^{\circ}(n=4-12),{ }^{3} F_{3}^{\circ}(n=4-9$, $11),{ }^{3} F_{4}^{\circ}(n=5-10),{ }^{1} F_{3}^{\circ}(n=5-7,10-11), 5 d 4 f(3 / 2,5 / 2)$, $5 d 4 f(3 / 2,7 / 2)_{1}$, and some $5 d 7 p$ and $5 d 8 p$ levels are listed in Table I. For each level in this table, the electronic configuration, the term, and the energy position were obtained from the same reference indicated by a superscript in column 1 . Most of them were obtained from the NIST atomic spectra database and the references therein [37]. The quoted error bars consist of both the statistical uncertainties from different recordings and the systematic errors resulting from the fitting procedure. Since the decay rates of unperturbed Rydberg levels are approximately proportional to $\left(n^{*}\right)^{3}$ with $n^{*}$ the effective principal quantum number [14], their lifetimes rapidly get longer as $n$ increases. Therefore, for the weakly perturbed levels with high $n$ values, the fluorescence emission is weak and difficult to detect. Moreover, the flightout-of-view effects and collision-induced quenching notably influence the lifetime measurements of such levels. So the measurements of lifetimes longer than $1 \mu$ s could not be performed.

The measured lifetime values are in the range from 11.3 to $901 \mathrm{~ns}$. Those of the $6 s 7 p^{1} P_{1}^{\circ}$ and $6 s 8 p^{1} P_{1}^{\circ}$ levels are in good agreement with the previous results measured by the Hanle effect method [27,29] and the LIF technique [20], respectively. However, for the $6 s 7 f^{1} F_{3}^{\circ}$ and $6 s 7 p^{3} P_{1}^{\circ}$ levels, our results are slightly longer than the previous measurements based on the super-radiant population of the excited levels [18] and obtained by the Hanle method [29], respectively. In Ref. [18], though the super-radiant effect has been suppressed in a cavity, due 
TABLE I. Measured lifetimes of odd-parity levels in the $6 s n p, 6 s n f, 5 d 4 f$, and $5 d n p$ configurations of Ba I.

\begin{tabular}{|c|c|c|c|c|c|c|c|}
\hline \multirow[b]{2}{*}{ Config. } & \multirow[b]{2}{*}{ Term } & \multirow[b]{2}{*}{ Level $\left(\mathrm{cm}^{-1}\right)$} & \multirow[b]{2}{*}{ Exc. $(\mathrm{nm})$} & \multirow[b]{2}{*}{ Obs. (nm) } & \multicolumn{3}{|c|}{ Lifetime (ns) } \\
\hline & & & & & $\begin{array}{c}\text { This work } \\
\text { Expt. }\end{array}$ & $\begin{array}{c}\text { This work } \\
\text { HFR }\end{array}$ & Others \\
\hline $6 s 7 p^{\mathrm{a}}$ & ${ }^{3} P_{1}^{\circ}$ & 30815.512 & 324.51 & 459.1 & $106(3)$ & 67.18 & $85.0(8.0)^{\mathrm{e}}$ \\
\hline $6 s 7 p^{\mathrm{a}}$ & ${ }^{1} P_{1}^{\circ}$ & 32547.033 & 307.25 & 425.3 & $12.6(4)$ & 10.52 & $\begin{array}{l}13.2(4)^{\mathrm{f}} \\
13.5(6)^{\mathrm{e}}\end{array}$ \\
\hline $6 s 4 f^{\mathrm{a}}$ & ${ }^{3} F_{2}^{\circ}$ & 34602.765 & 430.90 & 399.9 & $25.8(9)$ & 35.62 & \\
\hline $6 s 4 f^{\mathrm{a}}$ & ${ }^{3} F_{3}^{\circ}$ & 34616.643 & 430.64 & 399.7 & $26.6(1)$ & 35.18 & \\
\hline $6 s 4 f^{\text {a }}$ & ${ }^{1} F_{3}^{\circ}$ & 34736.373 & 428.43 & 428.4 & $26.8(1)$ & 30.06 & \\
\hline $6 s 8 p^{\mathrm{a}}$ & ${ }^{3} P_{0}^{\circ}$ & 35648.5 & 375.74 & 375.7 & $363(18)$ & 217.6 & \\
\hline $6 s 8 p^{\mathrm{a}}$ & ${ }^{3} P_{1}^{\circ}$ & 35669.00 & 375.45 & 412.0 & $338(14)$ & 202.2 & \\
\hline $6 s 8 p^{\mathrm{a}}$ & ${ }^{3} P_{2}^{\circ}$ & 35757 & 374.21 & 410.5 & 293(15) & 208.1 & \\
\hline $6 s 8 p^{\mathrm{a}}$ & ${ }^{1} P_{1}^{\circ}$ & 35892.465 & 372.32 & 408.2 & $144(4)$ & 126.1 & $136(2)^{\mathrm{g}}$ \\
\hline $5 d 7 p^{\mathrm{a}}$ & ${ }^{3} D_{1}^{\circ}$ & 36495.732 & 364.14 & 398.4 & $106(3)$ & 62.03 & \\
\hline $5 d 7 p^{\mathrm{a}}$ & ${ }^{3} F_{3}^{\circ}$ & 36511.207 & 366.36 & 398.2 & $137(4)$ & 101.6 & \\
\hline $5 d 7 p^{\mathrm{a}}$ & ${ }^{3} P_{0}^{\circ}$ & 36908.280 & 358.75 & 358.8 & $75(3)$ & 50.03 & \\
\hline $5 d 7 p^{\mathrm{a}}$ & ${ }^{3} P_{1}^{\circ}$ & 36989.981 & 360.04 & 270.4 & $65(2)$ & 42.31 & \\
\hline $5 d 7 p^{\mathrm{a}}$ & ${ }^{3} D_{2}^{\circ}$ & 37063.452 & 364.07 & 356.8 & $128(6)$ & 81.68 & \\
\hline $5 d 7 p^{\mathrm{a}}$ & ${ }^{3} P_{2}^{\circ}$ & 37077.477 & 363.89 & 389.4 & $84(4)$ & 52.81 & \\
\hline $5 d 7 p^{\mathrm{a}}$ & ${ }^{3} F_{4}^{\circ}$ & 37132.036 & 363.17 & 363.2 & $119(5)$ & 155.1 & \\
\hline $5 d 7 p^{\mathrm{a}}$ & ${ }^{1} F_{3}^{\circ}$ & 37282.124 & 361.20 & 386.3 & $81(2)$ & 97.98 & \\
\hline $6 s 5 f^{\mathrm{a}}$ & ${ }^{3} F_{2}^{\circ}$ & 37394.868 & 359.73 & 697.7 & $48(4)$ & 36.53 & \\
\hline $6 s 5 f^{\mathrm{a}}$ & ${ }^{3} F_{3}^{\circ}$ & 37418.920 & 359.42 & 384.7 & $43(4)$ & 34.87 & \\
\hline $6 s 5 f^{\mathrm{a}}$ & ${ }^{3} F_{4}^{\circ}$ & 37524.148 & 358.07 & 358.1 & $57(2)$ & $33.30^{\mathrm{k}}$ & \\
\hline $5 d 7 p^{\mathrm{a}}$ & ${ }^{3} D_{3}^{\circ}$ & 37540.184 & 357.86 & 353.0 & $92(3)$ & $62.48^{\mathrm{k}}$ & \\
\hline $6 s 5 f^{\mathrm{a}}$ & ${ }^{1} F_{3}^{\circ}$ & 37739.734 & 355.33 & 379.6 & $93(2)$ & $44.04^{\mathrm{k}}$ & \\
\hline $6 s 9 p^{\mathrm{a}}$ & ${ }^{1} P_{1}^{\circ}$ & 37775.28 & 347.93 & 379.1 & $255(10)$ & $71.81^{\mathrm{k}}$ & \\
\hline $6 s 9 p^{\mathrm{a}}$ & ${ }^{3} P_{1}^{\circ}$ & 37936.87 & 345.99 & 376.8 & $331(16)$ & 320.9 & \\
\hline $5 d 7 p^{\mathrm{a}}$ & ${ }^{1} P_{1}^{\circ}$ & 38499.860 & 341.48 & 569.3 & $39(4)$ & 45.42 & \\
\hline $6 s 6 f^{\mathrm{a}}$ & ${ }^{3} F_{2}^{\circ}$ & 38815.700 & 342.24 & 559.2 & $93(3)$ & 67.84 & \\
\hline $6 s 6 f^{\mathrm{a}}$ & ${ }^{3} F_{3}^{\circ}$ & 38819.378 & 342.20 & 337.8 & $68(2)$ & 67.49 & \\
\hline $6 s 6 f^{\mathrm{a}}$ & ${ }^{3} F_{4}^{\circ}$ & 38825.242 & 342.13 & 342.2 & $75(3)$ & 66.87 & \\
\hline $6 s 6 f^{\mathrm{a}}$ & ${ }^{1} F_{3}^{\circ}$ & 38883.903 & 341.44 & 363.8 & $91(4)$ & 46.79 & \\
\hline $6 s 10 p^{\mathrm{a}}$ & ${ }^{3} P_{1}^{\circ}$ & 39160.21 & 331.94 & 360.2 & $769(23)$ & 609.7 & \\
\hline $5 d 7 p^{\mathrm{d}}$ & ${ }^{3} F_{2}^{\circ}$ & 39266 & 330.78 & 545.5 & $40(2)$ & $63.16^{\mathrm{i}}$ & \\
\hline $6 s 7 f^{\mathrm{a}}$ & ${ }^{3} F_{2}^{\circ}$ & 39678.176 & 332.43 & 332.4 & $105(3)$ & 78.47 & \\
\hline $6 s 7 f^{\mathrm{a}}$ & ${ }^{3} F_{3}^{\circ}$ & 39680.727 & 332.40 & 332.4 & 101(4) & 97.23 & \\
\hline $6 s 7 f^{\mathrm{a}}$ & ${ }^{3} F_{4}^{\circ}$ & 39683.126 & 332.37 & 542.5 & $99(4)$ & 104.4 & \\
\hline $6 s 7 f^{\mathrm{a}}$ & ${ }^{1} F_{3}^{\circ}$ & 39705.106 & 327.98 & 353.2 & $91(2)$ & 59.76 & $64(6)^{\mathrm{h}}$ \\
\hline $5 d 4 f^{\text {a }}$ & $(3 / 2,5 / 2)_{1}$ & 39893.48 & 324.05 & 350.9 & $137(5)$ & 38.59 & \\
\hline $6 s 11 p^{\mathrm{b}}$ & ${ }^{3} P_{0}^{\circ}$ & 39902.44 & 323.96 & 324.0 & $756(21)$ & 754.5 & \\
\hline $6 s 11 p^{a}$ & ${ }^{3} P_{1}^{\circ}$ & 39916.35 & 323.81 & 350.6 & 131(6) & $737.0^{j}$ & \\
\hline $6 s 11 p^{\mathrm{a}}$ & ${ }^{3} P_{2}^{\circ}$ & 39930.79 & 323.66 & 350.4 & $128(5)$ & $801.9^{j}$ & \\
\hline $6 s 11 p^{\mathrm{a}}$ & ${ }^{1} P_{1}^{\circ}$ & 39982.14 & 323.12 & 349.8 & $118(5)$ & 83.55 & \\
\hline $6 s 8 f^{c}$ & ${ }^{3} F_{2}^{\circ}$ & 40239.076 & 322.34 & 346.7 & $335(11)$ & 175.0 & \\
\hline $6 s 8 f^{c}$ & ${ }^{3} F_{3}^{\circ}$ & 40241.571 & 326.32 & 346.7 & 113(4) & 152.5 & \\
\hline $6 s 8 f^{c}$ & ${ }^{3} F_{4}^{\circ}$ & 40244.293 & 326.29 & 326.3 & $156(3)$ & 161.5 & \\
\hline $6 s 12 p^{a}$ & ${ }^{3} P_{1}^{\circ}$ & 40395.60 & 320.72 & 344.8 & $878(32)$ & 701.5 & \\
\hline $6 s 12 p^{\mathrm{a}}$ & ${ }^{3} P_{2}^{\circ}$ & 40406.67 & 320.60 & 344.7 & $796(41)$ & 862.5 & \\
\hline $6 s 12 p^{a}$ & ${ }^{1} P_{1}^{\circ}$ & 40428.68 & 318.53 & 344.4 & $449(18)$ & 201.0 & \\
\hline $6 s 9 f^{\mathrm{a}}$ & ${ }^{3} F_{2}^{\circ}$ & 40613.87 & 322.40 & 342.2 & $105(4)$ & $322.3^{j}$ & \\
\hline $6 s 9 f^{c}$ & ${ }^{3} F_{3}^{\circ}$ & 40622.011 & 322.32 & 342.2 & $262(13)$ & 230.5 & \\
\hline $6 s 9 f^{c}$ & ${ }^{3} F_{4}^{\circ}$ & 40627.943 & 322.25 & 342.1 & 397(9) & 198.6 & \\
\hline $5 d 4 f^{\mathrm{a}}$ & $(3 / 2,7 / 2)_{1}$ & 40662.86 & 316.17 & 582.0 & $48(2)$ & 43.55 & \\
\hline $6 s 13 p^{\mathrm{a}}$ & ${ }^{1} P_{1}^{\circ}$ & 40765.23 & 316.96 & 340.5 & $267(11)$ & 93.53 & \\
\hline $5 d 8 p^{\mathrm{a}}$ & ${ }^{3} D_{1}^{\circ}$ & 40893.76 & 315.67 & 339.0 & $302(11)$ & $49.49^{k}$ & \\
\hline $6 s 10 f^{\mathrm{a}}$ & ${ }^{3} F_{2}^{\circ}$ & 40895.14 & 315.66 & 339.0 & $790(33)$ & 123.7 & \\
\hline $6 s 10 f^{c}$ & ${ }^{3} F_{4}^{\circ}$ & 40897.333 & 319.48 & 319.5 & $331(17)$ & 307.3 & \\
\hline $6 s 10 f^{\mathrm{b}}$ & ${ }^{1} F_{3}^{\circ}$ & 40898.912 & 315.62 & 338.9 & $283(11)$ & 231.9 & \\
\hline
\end{tabular}


TABLE I. (Continued.)

\begin{tabular}{lllcccc}
\hline \hline & & & & & & Lifetime (ns) \\
\cline { 5 - 6 } Config. & Term & Level $\left(\mathrm{cm}^{-1}\right)$ & Exc. $(\mathrm{nm})$ & Obs. $(\mathrm{nm})$ & $\begin{array}{c}\text { This work } \\
\text { Expt. }\end{array}$ & $\begin{array}{c}\text { This work } \\
\text { HFR }\end{array}$ \\
\hline $6 s 14 p^{\mathrm{a}}$ & ${ }^{3} P_{2}^{\circ}$ & 40982.86 & 318.61 & 338.0 & $901(48)$ & 1083 \\
$6 s 14 p^{\mathrm{a}}$ & ${ }^{1} P_{1}^{\circ}$ & 40991.23 & 314.71 & 337.9 & $576(21)$ & 189.6 \\
$5 d 8 p^{\mathrm{a}}$ & ${ }^{3} P_{0}^{\circ}$ & 41083.92 & 312.01 & 312.0 & $271(11)$ & \\
$6 s 11 f^{\mathrm{b}}$ & ${ }^{3} F_{4}^{\circ}$ & 41096.855 & 317.46 & 317.5 & $630(11)$ & \\
$5 d 8 p^{\mathrm{a}}$ & ${ }^{3} P_{1}^{\circ}$ & 41097.20 & 311.88 & 336.7 & $280(12)$ & \\
$6 s 11 f^{\mathrm{b}}$ & ${ }^{3} F_{3}^{\circ}$ & 41097.035 & 313.66 & 336.7 & $428(15)$ & \\
$6 s 11 f^{\mathrm{a}}$ & ${ }^{3} F_{2}^{\circ}$ & 41100.7 & 313.63 & 336.6 & $800(32)$ & \\
$6 s 11 f^{\mathrm{b}}$ & ${ }^{1} F_{3}^{\circ}$ & 41120.299 & 313.43 & 336.4 & $468(20)$ & \\
$6 s 12 f^{\mathrm{a}}$ & ${ }^{3} F_{2}^{\circ}$ & 41251.2 & 312.15 & 334.9 & $332(16)$ & \\
$6 s 16 f^{\mathrm{b}}$ & ${ }^{1} F_{3}^{\circ}$ & 41586.238 & 308.92 & 484.2 & $13.8(8)$ & \\
$5 d 8 p^{\mathrm{b}}$ & ${ }^{3} D_{3}^{\circ}$ & 41622.987 & 312.24 & 483.4 & $20.0(9)$ & \\
$6 s 22 p^{\mathrm{b}}$ & ${ }^{3} P_{2}^{\circ}$ & 41704.061 & 311.45 & 481.5 & $11.3(1)$ & \\
$5 d 8 p^{\mathrm{b}}$ & ${ }^{3} F_{4}^{\circ}$ & 41708.987 & 311.41 & 488.8 & $13.5(8)$ & \\
$5 d 8 p^{\mathrm{b}}$ & ${ }^{1} F_{3}^{\circ}$ & 41745.004 & 311.06 & 480.5 & $12.0(7)$ & \\
$5 d 8 p^{\mathrm{a}}$ & ${ }^{3} P_{2}^{\circ}$ & 41759.93 & 307.27 & 480.2 & $12.2(6)$ & \\
\hline \hline
\end{tabular}

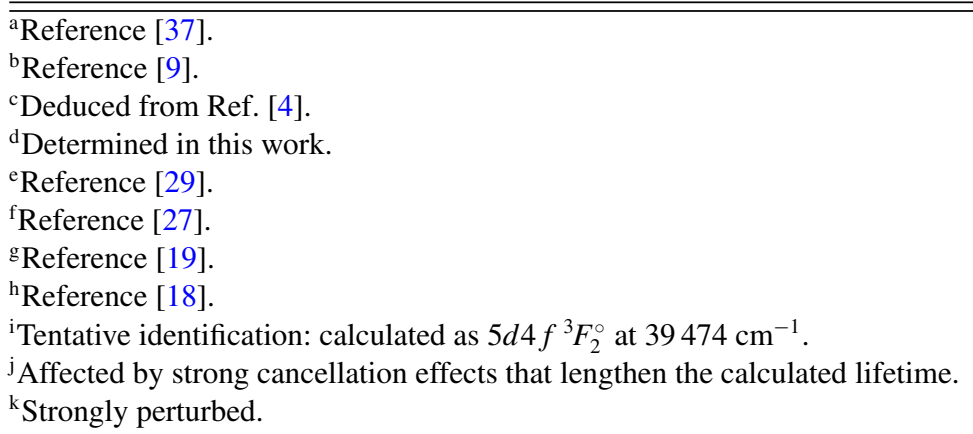

to the large atomic density $\left(10^{10} \mathrm{~cm}^{-3}\right)$ and accordingly the large atomic speed, it cannot be ensured that the collisions between the atoms had no effect on the lifetime measurements. Under the experimental conditions of Ref. [18], the collisional depopulation rate of the $6 s 7 f^{1} F_{3}^{\circ}$ level estimated by the relation $\Gamma^{\text {coll }}=\sigma_{n} \bar{v} N$ (where $\sigma_{n}$ is the collision cross section, $\bar{v}$ the mean relative velocity, and $N$ the collision density of partners) [38] was comparable to the total spontaneous radiative rate measured in the present work. The cross section $\sigma_{n}$, which scales as $n^{4}$ ( $n$ is the principal quantum number of the excited level), was simply estimated according to the experimental results for Cs atoms reported in Ref. [39]. So it is very likely that the residual collisions have shortened the $6 s 7 f^{1} F_{3}^{\circ}$ lifetime measured in Ref. [18]. The lifetime value measured by the Hanle effect method is inversely proportional to the half-width of the recorded Hanle curve. This method is very useful for reliable and accurate determination of short lifetimes. But for longer lifetimes, compared with the narrow natural linewidth, the contribution of the Doppler effect and of other possible broadenings to the width of the Hanle curve cannot be neglected and hence it also shortens the measured lifetime [29,40-42]. But Doppler broadening has no influence on LIF measurements. Therefore, it is reasonable to think that the lifetime values of the $6 s 7 f^{3} F_{3}^{\circ}$ and $6 s 7 p^{3} P_{1}^{\circ}$ levels measured in the present paper are more reliable than the previous measurements.

\section{THEORETICAL DETERMINATION OF LIFETIMES}

In order to compute the lifetimes of the high-lying members of the odd-parity Rydberg series $6 s n l(l=p, f$ and $n<20)$ along with those of the odd-parity perturber series $5 \mathrm{dnl}(n l=$ $7 p, 8 p, 4 f)$ measured in this work, we used the Hartree-Fock method with relativistic corrections (HFR) implemented in the Cowan computer suite [43] in which we have incorporated the core-polarization effects in the form a pseudopotential and a correction to the dipole operator, giving rise to the so-called HFR + CPOL method [44]. In the past, this method has appeared to be effective in predicting the radiative properties of heavy near-neutral and neutral atoms (see, e.g., Refs. [45-47] for review papers), although it could be challenging when considering perturbed Rydberg series of such species (see, e.g., Ref. [33]).

The core-polarization parameters, that is, the dipole polarizability of the ionic core, $\alpha_{d}$, and the cutoff radius $r_{c}$, corresponded to a $\mathrm{Ba}$ III core surrounded by two valence electrons. For the dipole polarizability, we chose the relativistic random-phase approximation value calculated for Ba III by Johnson et al. [48] (i.e., $\alpha_{d}=1.921$ a.u.). The HFR average radius of the $5 p$ orbital, the outermost core orbital, has been taken as the cutoff radius, $r_{c}=\langle 5 p|r| 5 p\rangle=10.61$ a.u. The valence-valence correlations of the two surrounding valence electrons have been considered through configuration interaction (CI) expansions. The following CI expansions 
TABLE II. Comparison of the Ba I theoretical HFR lifetimes obtained in the present work with previous experimental results.

\begin{tabular}{|c|c|c|c|c|}
\hline Level & $\begin{array}{l}\text { Energy } \\
\left(\mathrm{cm}^{-1}\right)\end{array}$ & $\begin{array}{c}\text { Previous } \\
\text { Expt. } \\
\text { (ns) }\end{array}$ & $\begin{array}{c}\text { This work } \\
\text { HFR } \\
\text { (ns) }\end{array}$ & $\begin{array}{c}\text { This work } \\
\text { Expt. } \\
\text { (ns) }\end{array}$ \\
\hline $6 s 6 p{ }^{1} P_{1}^{\circ}$ & 18060.261 & $\begin{array}{c}8.36(25)^{\mathrm{a}} \\
8.2(2)^{\mathrm{b}} \\
8.37(14)^{\mathrm{c}} \\
8.37(8)^{\mathrm{d}}\end{array}$ & 9.38 & \\
\hline $5 d 6 p^{3} D_{1}^{\circ}$ & 24192.033 & $\begin{array}{l}17.0(5)^{\mathrm{e}} \\
11.1(2.3)^{\mathrm{h}}\end{array}$ & 15.93 & \\
\hline $5 d 6 p^{3} D_{3}^{\circ}$ & 24979.834 & $10.2(1.5)^{\mathrm{g}}$ & 15.69 & \\
\hline $5 d 6 p^{3} P_{1}^{\circ}$ & 25704.110 & $11.7(9)^{\mathrm{f}}$ & 10.85 & \\
\hline $5 d 6 p^{1} F_{3}^{\circ}$ & 26816.266 & $44.5(1.8)^{\mathrm{h}}$ & 41.35 & \\
\hline $5 d 6 p^{1} P_{1}^{\circ}$ & 28554.221 & $\begin{array}{c}12.17(9)^{\mathrm{h}} \\
12.4(9)^{\mathrm{f}} \\
29(2)^{\mathrm{i}}\end{array}$ & 10.61 & \\
\hline $6 s 7 p^{1} P_{1}^{\circ}$ & 32547.033 & $\begin{array}{l}13.2(4)^{\mathrm{e}} \\
13.5(6\end{array}$ & 10.52 & $12.6(4)$ \\
\hline $6 s 4 f^{3} F_{2}^{\circ}$ & 34602.765 & $35(3)^{\mathrm{i}}$ & 35.62 & $25.8(9)$ \\
\hline $6 s 4 f^{3} F_{4}^{\circ}$ & 34630.779 & $31(3)^{\mathrm{i}}$ & 35.21 & \\
\hline $6 s 7 d^{3} D_{2}$ & 35616.949 & $33(2)^{j}$ & 36.18 & \\
\hline $6 s 7 d^{3} D_{1}$ & 35709.289 & $34(2)^{j}$ & 35.87 & \\
\hline $5 d 6 d^{3} D_{1}$ & 35933.806 & $28(5)^{j}$ & 22.65 & \\
\hline $5 d 6 d^{3} D_{2}$ & 36200.412 & $23(2)^{j}$ & 28.02 & \\
\hline $5 d 6 d^{3} S_{1}$ & 37095.486 & $25(2)^{j}$ & 22.96 & \\
\hline $6 s 8 d^{1} D_{2}$ & 37435.176 & $20.1(8)^{\mathrm{g}}$ & $39.56^{1}$ & \\
\hline $6 s 9 d^{1} D_{2}$ & 39335.003 & $25.5(1.4)^{\mathrm{g}}$ & $15.59^{\mathrm{m}}$ & \\
\hline $6 s 10 d^{1} D_{2}$ & 39998.35 & $43.3(1.4)^{\mathrm{g}}$ & $26.33^{\mathrm{m}}$ & \\
\hline $6 s 11 d^{1} D_{2}$ & 40483.59 & $102(3)^{\mathrm{g}}$ & 118.2 & \\
\hline $6 s 12 d^{1} D_{2}$ & 40781.43 & $\begin{array}{l}126(10)^{g} \\
139(6)^{\mathrm{k}}\end{array}$ & $89.89^{\mathrm{m}}$ & \\
\hline
\end{tabular}

\begin{tabular}{l}
\hline a Reference [24]. \\
${ }^{\mathrm{b}}$ Reference [25]. \\
${ }^{\mathrm{c}}$ Reference [26]. \\
${ }^{\mathrm{d}}$ Reference [50]. \\
${ }^{\mathrm{e}}$ Reference [27]. \\
${ }_{\mathrm{f}}^{\mathrm{f}}$ Reference [29]. \\
${ }^{\mathrm{g}}$ Reference [11]. \\
${ }^{\mathrm{h}}$ Reference [28]. \\
${ }_{\mathrm{i}}^{\mathrm{i}}$ Reference [51]. \\
j Reference [21]. \\
${ }^{\mathrm{k}}$ Reference [15]. \\
${ }^{\mathrm{l}}$ Affected by strong cancellation effects that lengthen the calculated \\
lifetime. \\
${ }^{\mathrm{m}}$ Strongly perturbed.
\end{tabular}

have been retained after several tests: $6 s^{2}+6 s n s+6 s n d+$ $6 s n^{\prime} g+5 d n^{\prime \prime} s+5 d n^{\prime \prime} d+6 p^{2}+6 p 4 f+4 f^{2}$ with $n \leqslant 19$, $n^{\prime} \leqslant 15$, and $n^{\prime \prime}<9$ for the even parity; $6 s n p+6 s n f+$ $5 d n^{\prime} p+5 d 4 f$ with $n \leqslant 19$ and $n^{\prime} \leqslant 9$ for the odd parity. In addition to the core-polarization effects and the CI expansions, a least-squares fitting procedure has been applied based upon the experimental energy levels compiled recently by Curry [49]. The standard deviations of the fits were 150 and $134 \mathrm{~cm}^{-1}$ for the even and odd parities, respectively.
The calculated lifetimes are reported in Table I along with our measurements. We also give a comparison of this calculation with other measurements found in the literature in Table II. Theoretical values, which are affected by strong cancellation effects, are marked with an asterisk. These effects reduce artificially the line strengths, calculated as squares of a sum of transition amplitudes that can be positive or negative [43], of important decay channels (these effects are strong when the cancellation factor as defined by Cowan [43] is less than or of the order of 0.05) and therefore lengthen the theoretical lifetimes. Concerning the disagreements between the experimental and theoretical lifetimes observed for the levels situated close to the ionization limit (typically above $41000 \mathrm{~cm}^{-1} ; 42034.91 \mathrm{~cm}^{-1}$ [47]), they are probably due to the missing contributions to the CI expansions of higher members $(n>19)$ of the Rydberg series up to continuum and beyond. For this reason, as the theory-experiment agreement deteriorates for higher $n$ values, no results are given in the tables above $41000 \mathrm{~cm}^{-1}$. The determination of more accurate values for such states would require the consideration in the models of many additional configurations, which is beyond our computer capabilities. For the remaining theory-experiment discrepancies, some of them in Table I but all of them in Table II can be explained by a strong perturbation of a particular Rydberg series member; they are marked with a superscript (see table footnotes). One can argue that Table II concerns mostly the low-lying Rydberg series members. Besides, it was noticed that the lifetimes of these long-lived Rydberg states (with lifetimes of the order of $100 \mathrm{~ns}$ and more) can be extremely sensitive to small admixtures of the perturbers. To illustrate this point, we decreased by a few percent the admixtures of the $5 d 8 p^{3} F_{2}^{\circ},{ }^{3} D_{2}^{\circ}$ states that perturb the $6 s 10 f^{3} F_{2}^{\circ}$ Rydberg state (i.e., from $9 \%$ and $5 \%$ to $8 \%$ and $4 \%$, respectively); the calculated lifetime increased from 123.7 to $363.9 \mathrm{~ns}$, thus by a factor of 3 .

In a general way, these two tables show a general good theory-experiment agreement but they also illustrate the difficulty to reproduce, in an accurate way, the perturbations appearing along Rydberg series in a heavy neutral element like barium. Some of the discrepancies can be explained by cancellation effects affecting the line strengths. The remaining ones, particularly for high excitation levels, could certainly be eliminated by the consideration in the models of a much larger number of higher excitation configurations and of the interaction with the continuum. Some care must therefore be exercised when combining theoretical branching fractions with experimental lifetimes in order to deduce the relevant transition probabilities. Accurate experimental measurement of a limited number of such branching fractions (presently missing) would be welcome to assess, in a more accurate way, the theoretical model adopted in the present paper.

\section{CONCLUSION}

The experimental lifetimes of 70 high-lying odd-parity levels of $\mathrm{Ba} \mathrm{I}$ in the range 30815.512 to $41759.93 \mathrm{~cm}^{-1}$ have been determined using the time-resolved laser-induced fluorescence technique. To our best knowledge, among them, 67 lifetimes were measured for the first time. Comparison of experiment with theoretical lifetimes calculated with an HFR 
approach incorporating core-polarization effects illustrates the difficulties associated with the use of Cowan's codes for calculating radiative parameters for transitions involving strongly perturbed high-energy levels along the Rydberg series of heavy neutral atoms. The new lifetimes obtained in the present work will be helpful for the determination of transition probabilities in $\mathrm{Ba}$ I when accurate branching fractions become available.

\section{ACKNOWLEDGMENTS}

This work was supported by the National Natural Science Foundation of China (Contracts No. 10574056, No. 10974066, and No. 10978012) and by the Program for New Century Excellent Talents in University (China) (Contract No. NCET05-0302). Financial support from the Belgian FRS-FNRS is also acknowledged.
[1] D. LeBoeuf et al., Nature (London) 450, 533 (2007).

[2] V. V. Kazakov, S. V. Markova, and G. G. Petrash, Kvantovaya Elektron. (Moscow) 11, 949 (1984).

[3] W. Whaling, P. Hannaford, R. M. Lowe, É. Biémont, and N. Grevesse, Astron. Astrophys. 153, 109 (1985).

[4] J. L. Carlsten, T. J. McIlrath, and W. H. Parkinson, J. Phys. B 8, 38 (1975).

[5] M. Aymar, P. Camus, M. Dieulin, and C. Morillon, Phys. Rev. A 18, 2173 (1978).

[6] M. Aymar and O. Robaux, J. Phys. B 12, 531 (1979).

[7] P. Camus, M. Dieulin, and A. El Himdy, Phys. Rev. A 26, 379 (1982).

[8] B. H. Post, W. Hogervorst, and W. Vassen, Phys. Rev. A 29, 2989 (1984).

[9] B. H. Post, W. Vassen, W. Hogervorst, M. Aymar, and O. Robaux, J. Phys. B 18, 187 (1985).

[10] W. Vassen, E. Bente, and W. Hogervorst, J. Phys. B 20, 2383 (1987).

[11] D. Kaiser, P. Kulina, A. E. Livingston, H. H. Radloff, and S. Tudorache, Z. Phys. A 285, 111 (1978).

[12] P. Hafner and W. H. E. Schwarz, J. Phys. B 11, 2975 (1978).

[13] T. F. Gallagher, W. Sandner, and K. A. Safinya, Phys. Rev. A 23, 2969 (1981).

[14] M. Aymar, R. J. Champeau, C. Delsart, and J. C. Keller, J. Phys. B 14, 4489 (1981).

[15] K. Bhatia, P. Grafström, C. Levinson, H. Lundberg, L. Nilsson, and S. Svanberg, Z. Phys. A 303, 1 (1981).

[16] M. Aymar, P. Grafström, C. Levinson, H. Lundberg, and S. Svanberg, J. Phys. B 15, 877 (1982).

[17] T. F. Gallagher, R. Kachru, and N. H. Tran, Phys. Rev. A 26, 2611 (1982).

[18] C. Leonard and R. H. Rinkleff, Phys. Lett. A 112, 208 (1985).

[19] J. E. Smedley and D. F. Marran, Phys. Rev. A 47, 126 (1993).

[20] Y. Matsuo, T. Nakajima, T. Kobayashi, and M. Takami, Phys. Rev. A 59, 2071 (1999).

[21] C. H. Li and D. Budker, Phys. Rev. A 74, 012512 (2006).

[22] M. A. Zaki Ewiss and I. H. Al-Ahdali, Il Nuovo Cimento D 18, 1 (1996).

[23] D. Kułaga, J. Migdałek, and O. Bar, J. Phys. B 34, 4775 (2001).

[24] E. Hulpke, E. Paul, and W. Paul, Z. Phys. 177, 257 (1964).

[25] A. Lurio, Phys. Rev. 136, A376 (1964).
[26] L. O. Dickie and F. M. Kelly, Can. J. Phys. 48, 879 (1970).

[27] L. O. Dickie and F. M. Kelly, Can. J. Phys. 49, 1098 (1971).

[28] L. O. Dickie and F. M. Kelly, Can. J. Phys. 49, 2630 (1971).

[29] J. Brecht, J. Kowalski, G. Lidö, I. J. Ma, and G. zu Putlitz, Z. Phys. 264, 273 (1973).

[30] P. E. Jessop and F. M. Pipkin, Phys. Rev. A 20, 269 (1979).

[31] G. Brink, A. Glassman, and R. Gupta, Opt. Commun. 33, 17 (1980).

[32] U. Berzinsh, L. Caiyan, R. Zerne, S. Svanberg, and É. Biémont, Phys. Rev. A 55, 1836 (1997).

[33] Z. Dai, Z. Jiang, H. Xu, Z. Zhang, S. Svanberg, É. Biémont, P. H. Lefèbvre, and P. Quinet, J. Phys. B 36, 479 (2003).

[34] Z. G. Zhang, S. Svanberg, P. Quinet, P. Palmeri, and É. Biémont, Phys. Rev. Lett. 87, 273001 (2001).

[35] E. A. Den Hartog, M. T. Herd, J. E. Lawler, C. Sneden, J. J. Cowan, and T. C. Beers, Astrophys. J. 619, 639 (2005).

[36] R. Mayo, J. Campos, M. Ortiz, H. Xu, S. Svanberg, G. Malcheva, and K. Blagoev, Eur. Phys. J. D 40, 169 (2006).

[37] NIST Atomic Spectra Database, Version 3.0. [http://physics.nist.gov/cgi-bin/ASD/energy1.pl] (unpublished).

[38] W. Demtröder, Laser Spectroscopy (Springer, Heidelberg, Germany, 1996).

[39] Z. Feng, L. Zhang, J. Zhao, C. Li, and S. Jia, J. Phys. B 42, 145303 (2009).

[40] J. Larsson, Phys. Scr. 49, 173 (1994).

[41] H. Xu, Z. Sun, Z. Dai, Z. Jiang, P. Palmeri, P. Quinet, and É. Biémont, Astron. Astrophys. 452, 357 (2006).

[42] S. Y. Lee, Ph.D. thesis, Adelaide University, 2007.

[43] R. D. Cowan, The Theory of Atomic Structure and Spectra (University of California, Berkeley, 1981).

[44] P. Quinet, P. Palmeri, É. Biémont, M. M. McCurdy, G. Rieger, E. H. Pinnington, M. E. Wickliffe, and J. E. Lawler, Mon. Not. R. Astron. Soc. 307, 934 (1999).

[45] V. Fivet, P. Quinet, P. Palmeri, É. Biémont, and H. L. Xu, J. Electron Spectrosc. Relat. Phenom. 156, 250 (2007).

[46] É. Biémont and P. Quinet, Phys. Scr., T 105, 38 (2003).

[47] É. Biémont, Phys. Scr., T 119, 55 (2005).

[48] W. R. Johnson, D. Kolb, and K.-N. Huang, At. Data Nucl. Data Tables 28, 333 (1983)

[49] J. J. Curry, J. Phys. Chem. Ref. Data 33, 725 (2004).

[50] F. M. Kelly and M. S. Mathur, Can. J. Phys. 55, 83 (1977).

[51] R. Schaefer, J. Quant. Spectrosc. Radiat. Transfer 11, 499 (1971). 